\title{
Spatially resolved images of reactive ions in the Orion $\mathrm{Bar}^{\star, \star \star}$
}

\author{
Javier R. Goicoechea ${ }^{1}$, Sara Cuadrado ${ }^{1}$, Jérôme Pety ${ }^{2,3}$, Emeric Bron ${ }^{1,3}$, John H. Black ${ }^{4}$, \\ José Cernicharo ${ }^{1}$, Edwige Chapillon ${ }^{2,5}$, Asunción Fuente ${ }^{6}$, and Maryvonne Gerin ${ }^{3}$ \\ ${ }^{1}$ Instituto de Ciencias de Materiales de Madrid (CSIC), 28049 Madrid, Spain \\ e-mail: jr.goicoechea@icmm.csic.es \\ 2 Institut de Radioastronomie Millimétrique, 38406 Saint-Martin d'Hères, France \\ 3 LERMA, Obs. de Paris, PSL Research University, CNRS, Sorbonne Universiteés, 75005, UPMC Univ. Paris 06, ENS, France \\ ${ }^{4}$ Chalmers University of Technology, Onsala Space Observatory, 43992 Onsala, Sweden \\ 5 OASU/LAB-UMR5804, CNRS, Université Bordeaux, 33615 Pessac, France \\ ${ }^{6}$ Observatorio Astronómico Nacional (IGN). Apartado 112, 28803 Alcalá de Henares, Spain
}

Received 1 March 2017 / Accepted 21 April 2017

\begin{abstract}
We report high angular resolution $\left(4.9^{\prime \prime} \times 3.0^{\prime \prime}\right)$ images of reactive ions $\mathrm{SH}^{+}, \mathrm{HOC}^{+}$, and $\mathrm{SO}^{+}$toward the Orion Bar photodissociation region (PDR). We used ALMA-ACA to map several rotational lines at $0.8 \mathrm{~mm}$, complemented with multi-line observations obtained with the IRAM $30 \mathrm{~m}$ telescope. The $\mathrm{SH}^{+}$and $\mathrm{HOC}^{+}$emission is restricted to a narrow layer of $2^{\prime \prime}$ - to $10^{\prime \prime}$-width $(\approx 800$ to $4000 \mathrm{AU}$ depending on the assumed PDR geometry) that follows the vibrationally excited $\mathrm{H}_{2}^{*}$ emission. Both ions efficiently form very close to the $\mathrm{H} / \mathrm{H}_{2}$ transition zone, at a depth of $A_{\mathrm{V}} \lesssim 1$ mag into the neutral cloud, where abundant $\mathrm{C}^{+}, \mathrm{S}^{+}$, and $\mathrm{H}_{2}^{*}$ coexist. $\mathrm{SO}^{+}$peaks slightly deeper into the cloud. The observed ions have low rotational temperatures $\left(T_{\text {rot }} \approx 10-30 \mathrm{~K} \ll T_{\mathrm{k}}\right)$ and narrow line-widths $\left(\sim 2-3 \mathrm{~km} \mathrm{~s}^{-1}\right)$, a factor of $\simeq 2$ narrower that those of the lighter reactive ion $\mathrm{CH}^{+}$. This is consistent with the higher reactivity and faster radiative pumping rates of $\mathrm{CH}^{+}$compared to the heavier ions, which are driven relatively more quickly toward smaller velocity dispersion by elastic collisions and toward lower $T_{\text {rot }}$ by inelastic collisions. We estimate column densities and average physical conditions from an excitation model $\left(n\left(\mathrm{H}_{2}\right) \approx 10^{5}-10^{6} \mathrm{~cm}^{-3}, n\left(\mathrm{e}^{-}\right) \approx 10 \mathrm{~cm}^{-3}\right.$, and $\left.T_{\mathrm{k}} \approx 200 \mathrm{~K}\right)$. Regardless of the excitation details, $\mathrm{SH}^{+}$and $\mathrm{HOC}^{+}$clearly trace the most exposed layers of the UV-irradiated molecular cloud surface, whereas $\mathrm{SO}^{+}$arises from slightly more shielded layers.
\end{abstract}

Key words. astrochemistry - line: identification - ISM: clouds - (ISM:) photon-dominated region (PDR)

\section{Introduction}

Reactive ions are transient species for which the timescale of reactive collisions with $\mathrm{H}_{2}, \mathrm{H}$, or $\mathrm{e}^{-}$(leading to a chemical reaction, and thus molecule destruction) is comparable to, or shorter than, that of inelastic collisions (Black 1998; Nagy et al. 2013; Godard \& Cernicharo 2013). The formation of reactive ions such as $\mathrm{CH}^{+}$and $\mathrm{SH}^{+}$depends on the availability of $\mathrm{C}^{+}$ and $\mathrm{S}^{+}$(i.e., of $\mathrm{UV}$ photons and thus high ionization fractions $x_{\mathrm{e}}=n\left(\mathrm{e}^{-}\right) / n_{\mathrm{H}}$ ), and on the presence of excited $\mathrm{H}_{2}$ (either UV-pumped or hot and thermally excited). This allows overcoming the high endothermicity (and sometimes energy barrier) of some of the key initiating chemical reactions (e.g., Gerin et al. 2016). The reaction $\mathrm{C}^{+}+\mathrm{H}_{2}(\mathrm{v}) \rightarrow \mathrm{CH}^{+}+\mathrm{H}$, for example, is endothermic by $\Delta E / k \simeq 4300 \mathrm{~K}$ if $v=0$, but exothermic and fast for $v \geq 1$ (Hierl et al. 1997; Agúndez et al. 2010). Despite their short lifetimes, reactive ions can be detected and used to probe energetic processes in irradiated, circumstellar (e.g.,

\footnotetext{
* This paper makes use of the following ALMA data: ADS/JAO.ALMA\#2012.1.00352.S. ALMA is a partnership of ESO (representing its member states), NSF (USA), and NINS (Japan), together with NRC (Canada), and NSC and ASIAA (Taiwan), in cooperation with the Republic of Chile. The Joint ALMA Observatory is operated by ESO, AUI/NRAO, and NAOJ.

$\star \star$ Includes IRAM $30 \mathrm{~m}$ telescope observations. IRAM is supported by INSU/CNRS (France), MPG (Germany), and IGN (Spain).
}

Cernicharo et al. 1997), interstellar (e.g., Fuente et al. 2003), or protostellar (e.g., Benz et al. 2016) gas.

$\mathrm{CO}^{+}$and $\mathrm{HOC}^{+}$(the metastable isomer of $\mathrm{HCO}^{+}$) have been detected in low angular resolution observations of UV-irradiated clouds near massive stars (Stoerzer et al. 1995; Apponi et al. 1999; Fuente et al. 2003). They are predicted to form close to the $\mathrm{H} / \mathrm{H}_{2}$ transition zone, the dissociation front (DF), by hightemperature reactions of $\mathrm{C}^{+}$with $\mathrm{OH}$ and $\mathrm{H}_{2} \mathrm{O}$, respectively (e.g., Sternberg \& Dalgarno 1995). $\mathrm{HOC}^{+}$also forms by the reaction $\mathrm{CO}^{+}+\mathrm{H}_{2} \rightarrow \mathrm{HOC}^{+}+\mathrm{H}$; thus, $\mathrm{CO}^{+}$and $\mathrm{HOC}^{+}$abundances are likely related (Smith et al. 2002). In photodissociation regions (PDRs), $\mathrm{SO}^{+}$is predicted to form primarily via the reaction $\mathrm{S}^{+}+\mathrm{OH} \rightarrow \mathrm{SO}^{+}+\mathrm{H}$ (e.g., Turner 1996). These ionneutral reactions leading to $\mathrm{CO}^{+}, \mathrm{HOC}^{+}$and $\mathrm{SO}^{+}$are highly exothermic.

Herschel allowed the detection of $\mathrm{OH}, \mathrm{CH}^{+}$, and $\mathrm{SH}^{+}$emission toward dense PDRs (Goicoechea et al. 2011; Nagy et al. 2013, 2017; Parikka et al. 2017). Unfortunately, the limited size of the space telescope did not permit us to resolve the $\Delta A_{V} \lesssim$ 1 mag extent of the DF (a few arcsec for the closest PDRs). Therefore, the true spatial distribution of the reactive ions emission is mostly unknown. Unlike $\mathrm{CH}^{+}$, rotational lines of $\mathrm{SH}^{+}$ can be observed from the ground (Müller et al. 2014). Here we report the first interferometric images of $\mathrm{SH}^{+}, \mathrm{HOC}^{+}$, and $\mathrm{SO}^{+}$.

The Orion Bar is a dense PDR (Hollenbach \& Tielens 1999) illuminated by a far-UV (FUV) $(6 \mathrm{eV}<E<13.6 \mathrm{eV})$ field of a 

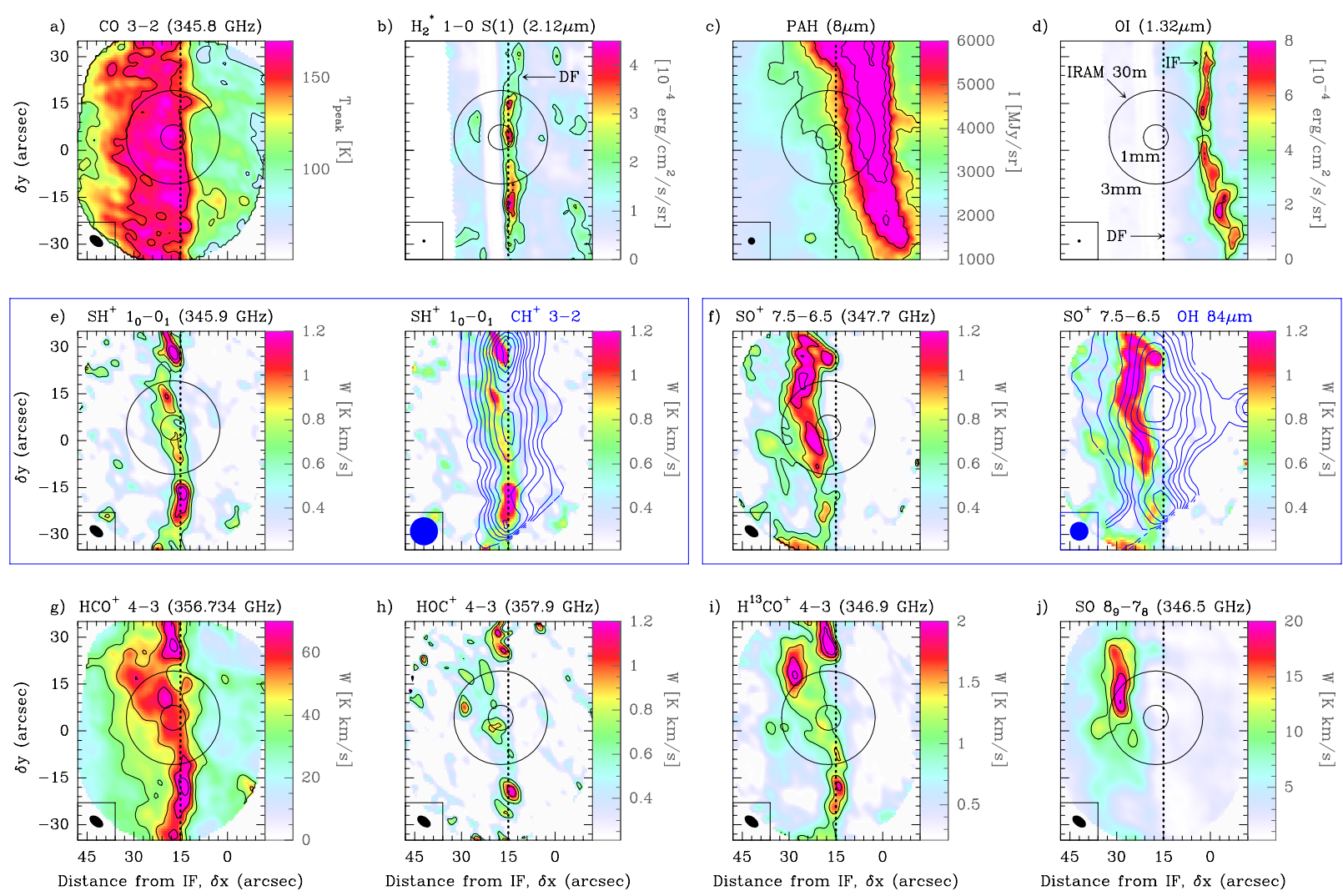

Fig. 1. ALMA-ACA and complementary images of the Bar. All images have been rotated to bring the FUV illuminating direction roughly in the horizontal direction (from the right). The circles indicate the position of our IRAM $30 \mathrm{~m}$ line survey, with beams of $\approx 8^{\prime \prime}\left(\approx 30^{\prime \prime}\right)$ at $1 \mathrm{~mm}(3 \mathrm{~mm})$. The upper row shows images of b) the $\mathrm{H}_{2} v=1-0 S(1)$ line at $2.12 \mu \mathrm{m}$, delineating the DF (Walmsley et al. 2000); c) the Spitzer $8 \mu \mathrm{m}$ emission produced mainly by PAHs (Megeath et al. 2012), and d) the fluorescent O I line at $1.32 \mu \mathrm{m}$ at the H II/PDR boundary (Walmsley et al. 2000). The blue contours in e) and f) represent the $\mathrm{CH}^{+} 119.8 \mu \mathrm{m}\left(\approx 9^{\prime \prime}\right.$ resolution) and $\mathrm{OH} 84.6 \mu \mathrm{m}\left(\approx 6^{\prime \prime}\right)$ lines mapped by Herschel (Parikka et al. 2017).

few $10^{4}$ times the mean interstellar radiation field. Because of its proximity ( $414 \mathrm{pc}$, Menten et al. 2007) and nearly edge-on orientation, the Bar is a template to investigate the dynamics and chemistry in strongly FUV-irradiated gas (e.g., Pellegrini et al. 2009; Cuadrado et al. 2015; Goicoechea et al. 2016).

\section{Observations and data reduction}

The interferometric images were taken with the $7 \mathrm{~m}$ antennas of the Atacama Compact Array (ACA), Chile. The observations consisted of a 10-pointing mosaic centered at $\alpha(2000)=5^{\mathrm{h}} 35^{\mathrm{m}} 20.6^{\mathrm{s}} ; \delta(2000)=-05^{\circ} 25^{\prime} 20^{\prime \prime}$. The total fieldof-view (FoV) is $\sim 50^{\prime \prime} \times 50^{\prime \prime}$. Target line frequencies lie in the $\sim 345-358 \mathrm{GHz}$ range (Table A.1). Lines were observed with correlators providing $\sim 500 \mathrm{kHz}$ resolution over a $937.5 \mathrm{MHz}$ bandwidth. The ALMA-ACA observation time was $\sim 6 \mathrm{~h}$. In order to recover the extended emission filtered out by the interferometer, we used fully sampled single-dish maps as zeroand short-spacings. The required high-sensitivity maps were obtained using the ALMA total-power $12 \mathrm{~m}$ antennas $\left(\sim 19^{\prime \prime}\right.$ resolution). We used the GILDAS/MAPPING software to create the short-spacing visibilities not sampled by ALMA-ACA. These visibilities were merged with the interferometric observations (Pety \& Rodríguez-Fernández 2010). The dirty image was deconvolved using the Högbom CLEAN algorithm. The resulting cubes were scaled from Jy/beam to brightness temperature scale using the synthesized beam size of $4.9^{\prime \prime} \times 3.0^{\prime \prime}$. The achieved rms noise is $\sim 10-20 \mathrm{mK}$ per $0.5 \mathrm{~km} \mathrm{~s}^{-1}$ smoothed channel, with an absolute flux accuracy of $\sim 10 \%$. The resulting images are shown in Fig. 1.

In addition, we carried out pointed observations toward the DF with the IRAM 30 m telescope (Spain). The observed position lies roughly at the center of the ALMA-ACA field (see circles in Fig. 1). This position is at $\triangle \mathrm{RA}=+3^{\prime \prime}$ and $\Delta \mathrm{Dec}=-3^{\prime \prime}$ from the "CO ${ }^{+}$peak" of Stoerzer et al. (1995). These multi-line observations are part of a complete $80-360 \mathrm{GHz}$ line survey at resolutions between $\sim 30^{\prime \prime}$ and $\sim 7^{\prime \prime}$ (Cuadrado et al. 2015).

\section{Results: morphology and emission properties}

In addition to the submillimeter (submm) emission images obtained with ALMA-ACA, panels b, c, and d in Fig. 1 show images of the DF traced by the vibrationally excited molecular hydrogen $\left(\mathrm{H}_{2}^{*}\right) v=1-0 S(1)$ line (Walmsley et al. 2000), of the atomic PDR (hydrogen is predominantly in neutral atomic form) as traced by the Spitzer/IRAC $8 \mu \mathrm{m}$ emission (Megeath et al. 2012), and of the ionization front (IF), the $\mathrm{H} / \mathrm{H}^{+}$transition zone. The $\delta x$ axis shows the distance in arcsec to the IF. Thus, in each panel, the FUV-photon flux decreases from right to left.

The peak of the optically thick CO 3-2 line provides a good lower limit to the gas temperature in the molecular PDR $(\delta x>$ $15^{\prime \prime}$ ), with $T_{\mathrm{k}} \gtrsim T_{\text {peak }}^{\mathrm{CO} 3-2} \simeq 150 \mathrm{~K}$ (Fig. 1a). The ALMA-ACA images show that, except for $\mathrm{SO}^{+}$, the emission from reactive ions starts very close to the DF, and globally follows that of $\mathrm{H}_{2}^{*}$. On small scales $\left(\lesssim 3^{\prime \prime} \approx 1000 \mathrm{AU}\right)$, several emission peaks of these ions coincide with the brightest $\mathrm{H}_{2}^{*} v=1-0 S(1)$ peaks (e.g., at $\delta y \simeq-18^{\prime \prime}$ ). Although the $\mathrm{SH}^{+}$and $\mathrm{HOC}^{+}$peaks at 
a)

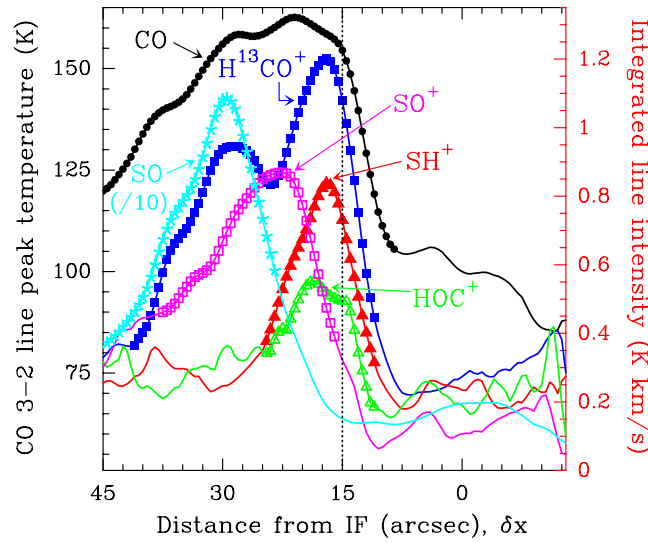

b)

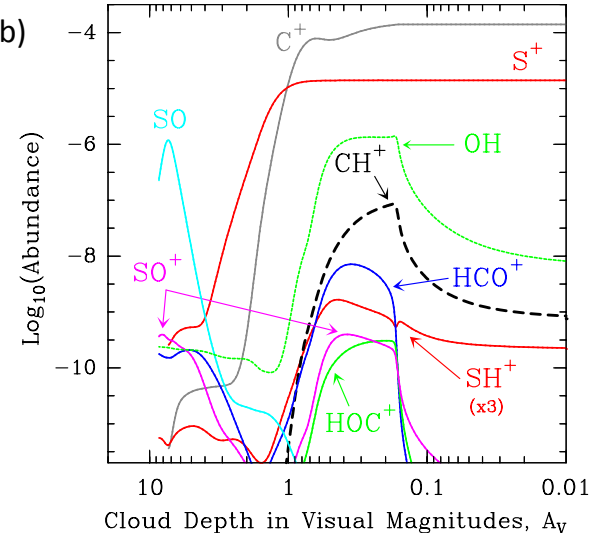

Fig. 2. a) ALMA-ACA averaged intensity cuts perpendicular to the Orion Bar. Except for CO $J=3-2$ line (peak temperature in K), cuts show integrated line intensities (in $\mathrm{K} \mathrm{km} \mathrm{s}^{-1}$ ). Symbols do not have any meaning, they are used to improve figure readability. b) Isobaric model of a PDR with $P_{\mathrm{th}} / k=2 \times 10^{8} \mathrm{~K} \mathrm{~cm}^{-3}$ and $\chi=10^{4}$. In both figures, the FUV radiation from the Trapezium cluster comes from the right-hand side.

$\delta y \simeq+27^{\prime \prime}$ do not exactly match a $\mathrm{H}_{2} v=1-0$ peak, observations do show the presence of extended $\mathrm{H}_{2} v=2-1$ and 1-0 emission along the $\mathrm{SH}^{+}$and $\mathrm{HOC}^{+}$-emitting zone (van der Werf et al. 1996). In fact, $\mathrm{H}_{2}^{*}$ emission from very high vibrational levels (up to $v=10$ or $E / k \approx 50000 \mathrm{~K}$ ) has recently been reported (Kaplan et al. 2017).

To investigate the molecular emission stratification, in Fig. 2a we show averaged emission cuts perpendicular to the Bar. The cuts demonstrate that the $\mathrm{SH}^{+} 1_{0}-0_{1}$ and $\mathrm{HOC}^{+}$4-3 lines arise from a narrow emission layer (akin to a filament), with a half-power-width of $\Delta(\delta x) \simeq 10^{\prime \prime}(\simeq 0.02 \mathrm{pc})$, that delineates the DF. The $\mathrm{H}^{13} \mathrm{CO}^{+} 4-3$ line displays this emission peak close to the DF, as well as another peak deeper inside the molecular cloud (at $\delta x \approx 30^{\prime \prime}$ ) that is dominated by emission from the colder molecular cloud interior. The $\mathrm{SO} 8_{9}-7_{8}$ line arises from these more FUV-shielded zones. These spatial emission trends are supported by the different line-widths (averaged over the ACA field of view, see Table A.1). In particular, the SO $8_{9}-78$ line is narrower $\left(\Delta v=1.8 \pm 0.1 \mathrm{~km} \mathrm{~s}^{-1}\right)$ than the $\mathrm{SO}^{+} 15 / 2^{-}-13 / 2^{+}\left(\Delta v=2.1 \pm 0.1 \mathrm{~km} \mathrm{~s}^{-1}\right), \mathrm{HOC}^{+} 4-3(\Delta v=$ $\left.2.7 \pm 0.1 \mathrm{~km} \mathrm{~s}^{-1}\right)$, and $\mathrm{SH}^{+} 1_{0}-0_{1}\left(\Delta v=2.8 \pm 0.1 \mathrm{~km} \mathrm{~s}^{-1}\right)$ lines that arise from the more FUV-irradiated gas near the molecular cloud edge.

We first derived $\mathrm{H}^{13} \mathrm{CO}^{+}, \mathrm{HOC}^{+}, \mathrm{SO}^{+}$, and $\mathrm{SO}$ rotational temperatures $\left(T_{\text {rot }}\right)$ and column densities $(N)$ by building rotational population diagrams from our IRAM $30 \mathrm{~m}$ observations (line survey position, Cuadrado et al. 2015). Results are shown in Tables 1 and B.1. $\mathrm{H}^{13} \mathrm{CO}^{+}$and $\mathrm{HOC}^{+}$have high dipole moments (and $\mathrm{SO}^{+}$to a lesser extent, but see Appendix D). Hence, the observed submm lines have moderate critical densities (several $10^{6} \mathrm{H}_{2} \mathrm{~cm}^{-3}$ ). The low- $J$ transitions toward the line survey position are subthermal $\left(T_{\text {rot }} \approx 15 \mathrm{~K} \ll T_{\mathrm{k}}\right)$. Their column densities are relatively small: from $\sim 10^{11} \mathrm{~cm}^{-2}$ (assuming uniform beam filling), to $\sim 10^{12} \mathrm{~cm}^{-2}$ (for a more realistic filamentary emission layer of $\sim 10^{\prime \prime}$ width and correcting for beam dilution).

In addition, we estimated the average physical conditions that lead to the $\mathrm{H}^{13} \mathrm{CO}^{+}, \mathrm{HOC}^{+}, \mathrm{SO}^{+}$and $\mathrm{SH}^{+}$emission toward the line survey position. We used a Monte Carlo model (Appendix D) that includes inelastic collisions with $\mathrm{H}_{2}$ and $\mathrm{e}^{-}$, as well as radiative excitation by the far-IR dust radiation field in the region (see Arab et al. 2012, and Fig. D.1). This allowed us to refine the source-averaged column density estimation for a $10^{\prime \prime}$-wide emission layer (Table 1). The observed $\mathrm{H}^{13} \mathrm{CO}^{+}, \mathrm{HOC}^{+}$, and $\mathrm{SO}^{+}$line intensities and $T_{\text {rot }}$ are reproduced with $n\left(\mathrm{H}_{2}\right) \simeq 10^{5} \mathrm{~cm}^{-3}, n\left(\mathrm{e}^{-}\right)=10 \mathrm{~cm}^{-3}$, and $T_{\mathrm{k}} \gtrsim 200 \mathrm{~K}$ (thus consistent with $T_{\text {peak }}^{\mathrm{CO}-2}$ ). However, with the set of assumed $\mathrm{SH}^{+}$collisional rates, fitting the $\mathrm{SH}^{+}$lines requires denser gas, $\sim 10^{6} \mathrm{~cm}^{-3}$ (see also Nagy et al. 2013). As $T_{\mathrm{k}}$ is expected to sharply vary along the DF (e.g., Sternberg \& Dalgarno 1995), the result of these single-component models should be taken as average conditions (over the ACA resolution). We note that at the distance to Orion, $4^{\prime \prime}$ is equivalent to $1 \mathrm{mag}$ of visual extinction for $n_{\mathrm{H}}=10^{5} \mathrm{~cm}^{-3}$.

Low $\left[\mathrm{HCO}^{+}\right] /\left[\mathrm{HOC}^{+}\right]$abundance ratios $(\sim 200-400)$ have previously been inferred from lower angular resolution $\left(10^{\prime \prime}\right.$ to $70^{\prime \prime}$, depending on the line and telescope) pointed observations toward the Bar (Apponi et al. 1999; Fuente et al. 2003). Such ratios are much lower than those predicted in FUV-shielded gas (>1000, e.g., Goicoechea et al. 2009). Given the similar $A_{\mathrm{ul}}$ coefficient and upper level energy $\left(E_{\mathrm{u}} / k\right)$ of the $\mathrm{H}^{13} \mathrm{CO}^{+}$and $\mathrm{HOC}^{+} 4-3$ transitions, their integrated intensity ratio is a good measure of the $\left[\mathrm{HCO}^{+}\right] /\left[\mathrm{HOC}^{+}\right]$isomeric ratio (lines are optically thin). The observed $\mathrm{H}^{13} \mathrm{CO}^{+} / \mathrm{HOC}^{+} 4-3$ line ratio is equivalent to a roughly constant $\left[\mathrm{HCO}^{+}\right] /\left[\mathrm{HOC}^{+}\right]$ratio of $145 \pm 5$ along the $\delta x=12-22^{\prime \prime}$ layer (assuming $\left[\mathrm{HCO}^{+}\right] /\left[\mathrm{H}^{13} \mathrm{CO}^{+}\right]=$ $67)$. The ratio then increases as the FUV-photon flux decreases, $\left[\mathrm{HCO}^{+}\right] /\left[\mathrm{HOC}^{+}\right]=180 \pm 4$ along the $\delta x=22-32^{\prime \prime}$ layer, until the $\mathrm{HOC}^{+} 4-3$ signal vanishes deeper inside the molecular cloud.

\section{Discussion}

$\mathrm{ALMA} \mathrm{HCO}^{+} 4-3$ images at $\sim 1^{\prime \prime}$ resolution suggest the presence of high-pressure structures, $P_{\mathrm{th}} / k=n_{\mathrm{H}} T_{\mathrm{k}} \approx(1-2) \times$ $10^{8} \mathrm{~K} \mathrm{~cm}^{-3}$, close to the DF (Goicoechea et al. 2016). Despite the different resolutions, several of these $\mathrm{HCO}^{+}$peaks coincide with the brightest $\mathrm{SH}^{+} 1_{0}-0_{1}$ and $\mathrm{OH} 84 \mu \mathrm{m}$ peaks (Figs. 1e and $\mathrm{f}$ ). Thus, it is reasonable to assume that $\mathrm{SH}^{+}$arises from these structures.

We used version 1.5.2 of the Meudon PDR code (Le Petit et al. 2006) to model the formation and destruction of reactive ions in a constant thermal-pressure slab of FUVirradiated gas $\left(P_{\mathrm{th}} / k\right.$ from $2 \times 10^{7}$ to $\left.2 \times 10^{8} \mathrm{~K} \mathrm{~cm}^{-3}\right)$. Figure $2 \mathrm{~b}$ shows abundance profiles (with respect to $\mathrm{H}$ nuclei) predicted by the high-pressure model. The FUV-photon flux is $\chi=10^{4}$ (in Draine units), the expected radiation field close to the DF. Compared to Nagy et al. (2013), we have included more recent rates for reactions of $\mathrm{S}^{+}$with $\mathrm{H}_{2}(v)$ (Zanchet et al. 2013). We adopt an undepleted sulfur abundance of $[\mathrm{S}]=1.4 \times 10^{-5}$ (Asplund et al. 2005). The predicted abundance stratification in 
Table 1. Inferred column densities and PDR model predictions.

\begin{tabular}{cccc}
\hline \hline & $\begin{array}{c}\log _{10} N\left[\mathrm{~cm}^{-2}\right] \\
\text { Rotational diagram }\end{array}$ & Non-LTE & \\
\hline $\mathrm{H}^{13} \mathrm{CO}^{+}$ & $11.8^{a}-12.1^{b}$ & 12.3 & $11.0^{c}-12.2^{d}$ \\
$\mathrm{HOC}^{+}$ & $11.6^{a}-11.9^{b}$ & 12.0 & $11.7^{c}-12.9^{d}$ \\
$\mathrm{SO}^{+}$ & $12.3^{a}-12.5^{b}$ & 12.5 & $12.4^{c}-13.5^{d}$ \\
$\mathrm{SH}^{+}$ & - & 13.2 & $11.7^{c}-12.8^{d}$ \\
\hline
\end{tabular}

Notes. ${ }^{(\dagger)}$ From a non-LTE model. ${ }^{(\ddagger)}$ Isobaric PDR model $\left(P_{\mathrm{th}} / k=\right.$ $2 \times 10^{8} \mathrm{~K} \mathrm{~cm}^{-3}$ and $\left.\chi=10^{4}\right)$. Columns integrated up to $A_{V}=10 .{ }^{(a)} \mathrm{As}-$ suming uniform beam filling. ${ }^{(b)}$ For a filament of $10^{\prime \prime}$-width. ${ }^{(c)}$ Face-on geometry. ${ }^{(d)}$ Edge-on geometry with a tilt angle of $\alpha \simeq 4^{\circ}$.

Fig. 2b qualitatively agrees with the observational intensity cut in Fig. 2a (but recall that the spatial scales are not directly comparable, as for the studied range of $P_{\mathrm{th}} / k$ in these 1D isobaric models, 10 mag of visual extinction corresponds to $\left.1-10^{\prime \prime}\right)$. To be more specific, we compared the inferred column densities with those predicted by the PDR model (Table 1). Because the Orion Bar is not a perfect edge-on PDR, this comparison requires a knowledge of the tilt angle $(\alpha)$ with respect to a pure edgeon geometry, and of the line-of-sight cloud-depth $\left(l_{\text {depth }}\right)$. Recent studies constrain $\alpha$ and $l_{\text {depth }}$ to $\lesssim 4^{\circ}$ and $\simeq 0.28 \mathrm{pc}$, respectively (Salgado et al. 2016; Andree-Labsch et al. 2017). For this geometry, the intrinsic width of the $\mathrm{SH}^{+}$- and $\mathrm{HOC}^{+}$-emitting layer would be narrower, from $\simeq 10^{\prime \prime}$ (the observed value) to $\simeq 2^{\prime \prime}$ (if the Bar is actually tilted). Given these uncertainties, the agreement between the range of observed and predicted columns is satisfactory for $\mathrm{HOC}^{+}, \mathrm{H}^{13} \mathrm{CO}^{+}$, and $\mathrm{SO}^{+}$(Table 1). Although PDR models with lower pressures predict qualitatively similar stratification, and a reactive ion abundance peak also at $A_{\mathrm{V}} \lesssim$ 1 mag (where $\mathrm{C}^{+}, \mathrm{S}^{+}$, and $\mathrm{H}_{2}^{*}$ coexist), a model with ten times lower $P_{\text {th }}$ underestimates $N\left(\mathrm{HOC}^{+}\right)$and $N\left(\mathrm{SO}^{+}\right)$by large factors $(\gtrsim 20)$. This is related to the lower predicted $\mathrm{OH}$ column densities (by a factor of $\sim 25$ ), key in the formation of $\mathrm{CO}^{+}, \mathrm{HOC}^{+}$and $\mathrm{SO}^{+}$(Goicoechea et al. 2011). Interestingly, $\mathrm{SO}^{+}$peaks deeper inside the cloud, while in the PDR models the main $\mathrm{SO}^{+}$peak is close to that of $\mathrm{SH}^{+}$. Compared to the other ions, $\mathrm{SO}^{+}$destruction near the DF is dominated by recombination and photodissociation (not by reactions with $\mathrm{H}_{2}$ or $\mathrm{H}$ ). Hence, $\left[\mathrm{SO}^{+}\right]$depends on the $[\mathrm{OH}] / x_{\mathrm{e}}$ ratio (Turner 1996) and on the photodissociation rate. The $\mathrm{SO}^{+}$line peaks deeper inside the cloud than the $\mathrm{OH}^{2} \Pi_{3 / 2} J=7 / 2^{-}-5 / 2^{+}$line (Fig. 1f) suggesting that near the DF either $n\left(\mathrm{e}^{-}\right)$is higher than in the model or, more likely, that the $\mathrm{SO}^{+}$photodissociation rate is larger. Indeed, $\mathrm{SO}^{+}$can be dissociated by lower $E$ photons ( $<5 \mathrm{eV}$, Bissantz et al. 1992). This process is not taken into account in the PDR model.

For $\mathrm{SH}^{+}$, we infer a column density that is a factor $\simeq 3-30$ above the PDR model prediction (depending on $\alpha$ ). Recall that reaction $\mathrm{S}^{+}+\mathrm{H}_{2}(v) \rightarrow \mathrm{SH}^{+}+\mathrm{H}$ (endothermic by $\Delta E / k \simeq$ $9860 \mathrm{~K}$ when $v=0$ ) only becomes exothermic when $v \geq 2$, but remains slow even then (Zanchet et al. 2013). The mismatch between model and observation, if relevant, may suggest an additional source of $\mathrm{SH}^{+}$that is not well captured by the model: overabundant $\mathrm{H}_{2}(v \geq 2)$ or temperature/pressure spikes due to the PDR dynamics (Goicoechea et al. 2016).

Regardless of the excitation details, our observations show that detecting $\mathrm{SH}^{+}$and $\mathrm{HOC}^{+}$emission is an unambiguous indication of FUV-irradiated gas. Intriguingly, $\mathrm{SH}^{+}$and $\mathrm{HOC}^{+}$linewidths are narrower than those of $\mathrm{CH}^{+}\left(\Delta v \simeq 4.5-5.5 \mathrm{~km} \mathrm{~s}^{-1}\right.$, Nagy et al. 2013; Parikka et al. 2017). The broader $\mathrm{CH}^{+}$lines in the Bar have been interpreted as a signature of $\mathrm{CH}^{+}$high reactivity (Nagy et al. 2013). In this view, the exothermicity (equivalent to an effective formation temperature of about $5360 \mathrm{~K}$ ) of the dominant formation route, reaction $\mathrm{C}^{+}+\mathrm{H}_{2}(v \geq 1) \rightarrow$ $\mathrm{CH}^{+}+\mathrm{H}$, goes into $\mathrm{CH}^{+}$excitation and motion. However, reactive collisions of $\mathrm{CH}^{+}$with $\mathrm{H}$ and $\mathrm{H}_{2}$ are faster than elastic and rotationally inelastic collisions (see Appendices $\mathrm{C}$ and $\mathrm{D}$ ). In particular, the $\mathrm{CH}^{+}$lifetime is so short (a few hours) that the molecule does not have time to thermalize, by elastic collisions, its translational motions to a velocity distribution at $T_{\mathrm{k}}$ (Black 1998). Hence, the broad lines would be related to the energy excess upon $\mathrm{CH}^{+}$formation (thousands of $\mathrm{K}$ ) and not to the actual $T_{\mathrm{k}}$ (at $T_{\mathrm{k}}=1000 \mathrm{~K}$, the $\mathrm{CH}^{+}$thermal line-width will only be $1.8 \mathrm{~km} \mathrm{~s}^{-1}$, Nagy et al. 2013), nor to an enhanced gas turbulence. Detailed models of the $\mathrm{CH}^{+}$excitation show that inclusion of formation and destruction rates in the level population determination affects the high- $J$ levels (Godard \& Cernicharo 2013). Indeed, using the $\mathrm{CH}^{+} J=3-2$ to $6-5$ intensities measured by Herschel/PACS toward the line survey position (Nagy et al. 2013), we derive $T_{\text {rot }}^{\mathrm{PACS}} \simeq 150 \mathrm{~K}$. This is significantly warmer than $T_{\text {rot }}$ of $\mathrm{SH}^{+}, \mathrm{HOC}^{+}$and $\mathrm{SO}^{+}$.

$\mathrm{CH}^{+}$is a light hydride (see Gerin et al. 2016, for a review) meaning that its lowest rotational transitions lie at far-IR frequencies. Therefore, its critical densities are very high $\left(n_{\mathrm{cr}}\left(\mathrm{CH}^{+}\right.\right.$ $4-3) \simeq 7 \times 10^{9} \mathrm{H}_{2} \mathrm{~cm}^{-3}$, cf. $n_{\mathrm{cr}}\left(\mathrm{HOC}^{+} 4-3\right) \simeq 4 \times 10^{6} \mathrm{H}_{2} \mathrm{~cm}^{-3}$ at $\left.T_{\mathrm{k}}=200 \mathrm{~K}\right)$. Such values are much higher than the gas density in the Orion Bar. Thus, without an excitation mechanism other than collisions, one would expect $T_{\text {rot }}\left(\mathrm{CH}^{+}\right) \ll$ $T_{\text {rot }}\left(\mathrm{HOC}^{+}\right)$. However, $\mathrm{CH}^{+}$has a shorter lifetime than the other ions, and shorter than the timescale for inelastic collisional excitations. Hence, the higher $T_{\text {rot }}^{\mathrm{PACS}}\left(\mathrm{CH}^{+}\right)$compared to the heavier ions must be related to a formation, and perhaps radiative, pumping mechanism. In particular, the dust continuum emission is much stronger in the far-IR than in the (sub)mm (Fig. D.1). As a consequence, the far-IR $\mathrm{CH}^{+}$rotational transitions have larger radiative pumping rates than the (sub)mm transitions of the heavier ions. As an example, we derive $B_{3 \rightarrow 4} U_{3 \rightarrow 4}^{\text {Dust+CMB }}\left(\mathrm{CH}^{+}\right) / B_{3 \rightarrow 4} U_{3 \rightarrow 4}^{\text {Dust+CMB }}\left(\mathrm{HOC}^{+}\right) \approx 100$ (where $B_{\text {lu }}$ is the stimulated absorption coefficient and $U$ is the energy density produced by the dust emission and by the cosmic background; see Appendix D.1). Therefore, $\mathrm{CH}^{+}$can be excited by radiation many times during its short lifetime (and during its mean-free-time for elastic collisions) so that it can remain kinetically hot (large velocity dispersion) and rotationally warm while it emits. On the other hand, the heavier ions are driven relatively more quicky toward smaller velocity dispersion by elastic collisions (narrower lines) and toward lower $T_{\text {rot }}$ by inelastic collisions. For $\mathrm{HOC}^{+}$, we estimate that several collisional excitations take place during its lifetime (several hours). This subtle but important difference likely explains the narrower lines and lower $T_{\text {rot }}$ of the heavier reactive ions, as well as their slightly different spatial distribution compared to $\mathrm{CH}^{+}$(see Fig. 1e).

Acknowledgements. We thank A. Parikka for sharing her Herschel far-IR $\mathrm{CH}^{+}$ and $\mathrm{OH}$ line maps. We thank the ERC for funding support under grant ERC-2013-Syg-610256-NANOCOSMOS, and the Spanish MINECO under grants AYA2012-32032, AYA2016-75066-C2-(1/2)-P and CSD2009-00038.

\section{References}

Agúndez, M., Goicoechea, J. R., Cernicharo, J., Faure, A., \& Roueff, E. 2010, ApJ, 713, 662

Andree-Labsch, S., Ossenkopf-Okada, V., \& Röllig, M. 2017, A\&A, 598, A2 Apponi, A. J., Pesch, T. C., \& Ziurys, L. M. 1999, ApJ, 519, L89

Arab, H., Abergel, A., Habart, E., et al. 2012, A\&A, 541, A19 
Asplund, M., Grevesse, N., \& Sauval, A. J. 2005, in Cosmic Abundances as Records of Stellar Evolution and Nucleosynthesis, eds. T. G. Barnes, III, \& F. N. Bash, ASP Conf. Ser., 336, 25

Benz, A. O., Bruderer, S., van Dishoeck, E. F., et al. 2016, A\&A, 590, A105

Bissantz, H., Andric, L., Hertzler, C., Foth, H.-J., \& Linder, F. 1992, Z. Phys. D Atoms Molecules Clusters, 22, 727

Black, J. H. 1998, Faraday Discussions, 109, 257

Cernicharo, J., Liu, X.-W., González-Alfonso, E., et al. 1997, ApJ, 483, L65

Cuadrado, S., Goicoechea, J. R., Pilleri, P., et al. 2015, A\&A, 575, A82

Flower, D. R. 1999, MNRAS, 305, 651

Fuente, A., Rodríguez-Franco, A., García-Burillo, S., Martín-Pintado, J., \& Black, J. H. 2003, A\&A, 406, 899

Fuente, A., García-Burillo, S., Usero, A., et al. 2008, A\&A, 492, 675

Gerin, M., Neufeld, D. A., \& Goicoechea, J. R. 2016, ARA\&A, 54, 181

Godard, B., \& Cernicharo, J. 2013, A\&A, 550, A8

Goicoechea, J. R., Pety, J., Gerin, M., et al. 2006, A\&A, 456, 565

Goicoechea, J. R., Pety, J., Gerin, M., et al. 2009, A\&A, 498, 771

Goicoechea, J. R., Joblin, C., Contursi, A., et al. 2011, A\&A, 530, L16

Goicoechea, J. R., Pety, J., Cuadrado, S., et al. 2016, Nature, 537, 207

Hierl, P. M., Morris, R. A., \& Viggiano, A. A. 1997, J. Chem. Phys., 106, 10145

Hollenbach, D. J., \& Tielens, A. G. G. M. 1999, Rev. Mod. Phys., 71, 173

Kaplan, K. F., Dinerstein, H. L., Oh, H., et al. 2017, ApJ, 838, 2
Le Petit, F., Nehmé, C., Le Bourlot, J., \& Roueff, E. 2006, ApJS, 164, 506 Lique, F., Spielfiedel, A., \& Cernicharo, J. 2006, A\&A, 451, 1125

Megeath, S. T., Gutermuth, R., Muzerolle, J., et al. 2012, AJ, 144, 192

Menten, K. M., Reid, M. J., Forbrich, J., \& Brunthaler, A. 2007, A\&A, 474, 515

Müller, H. S. P., Goicoechea, J. R., Cernicharo, J., et al. 2014, A\&A, 569, L5

Nagy, Z., Choi, Y., Ossenkopf-Okada, V., et al. 2017, A\&A, 599, A22

Nagy, Z., Van der Tak, F. F. S., Ossenkopf, V., et al. 2013, A\&A, 550, A96

Parikka, A., Habart, E., Bernard-Salas, J., et al. 2017, A\&A, 599, A20

Pellegrini, E. W., Baldwin, J. A., Ferland, G. J., Shaw, G., \& Heathcote, S. 2009, ApJ, 693, 285

Pety, J., \& Rodríguez-Fernández, N. 2010, A\&A, 517, A12

Salgado, F., Berné, O., Adams, J. D., et al. 2016, ApJ, 830, 118

Smith, M. A., Schlemmer, S., von Richthofen, J., \& Gerlich, D. 2002, ApJ, 578, L87

Sternberg, A., \& Dalgarno, A. 1995, ApJS, 99, 565

Stoerzer, H., Stutzki, J., \& Sternberg, A. 1995, A\&A, 296, L9

Turner, B. E. 1996, ApJ, 468, 694

van der Werf, P. P., Stutzki, J., Sternberg, A., \& Krabbe, A. 1996, A\&A, 313, 633

Walmsley, C. M., Natta, A., Oliva, E., \& Testi, L. 2000, A\&A, 364, 301

Zanchet, A., Agúndez, M., Herrero, V. J., Aguado, A., \& Roncero, O. 2013, AJ, 146,125 


\section{Appendix A: Spectroscopic parameters of the observed lines}

Table A.1. Frequencies, energy levels, Einstein coefficients and line parameters of the ALMA-ACA spectra averaged over the observed field.

\begin{tabular}{lcccccccc}
\hline \hline Species & Transition & $\begin{array}{c}\text { Frequency } \\
(\mathrm{MHz})\end{array}$ & $\begin{array}{c}E_{\mathrm{u}} / k \\
(\mathrm{~K})\end{array}$ & $\begin{array}{c}A_{\mathrm{ul}} \\
\left(\mathrm{s}^{-1}\right)\end{array}$ & $\begin{array}{c}\int T \mathrm{~d} v \\
\left(\mathrm{~K} \mathrm{~km} \mathrm{~s}^{-1}\right)\end{array}$ & $\begin{array}{c}v_{\text {peak,LSR }} \\
\left(\mathrm{km} \mathrm{s}^{-1}\right)\end{array}$ & $\begin{array}{c}\Delta v \\
\left(\mathrm{~km} \mathrm{~s}^{-1}\right)\end{array}$ & $\begin{array}{c}T_{\text {peak }} \\
(\mathrm{K})\end{array}$ \\
\hline $\mathrm{CO}$ & $3-2$ & 345795.9899 & 33.2 & $2.50 \times 10^{-6}$ & $625.67(0.72)$ & $10.1(0.1)$ & $4.6(0.1)$ & 127.41 \\
$\mathrm{HCO}^{+}$ & $4-3$ & 356734.2246 & 42.8 & $3.55 \times 10^{-3}$ & $33.93(0.25)$ & $10.5(0.1)$ & $3.1(0.1)$ & 10.41 \\
$\mathrm{H}^{13} \mathrm{CO}^{+}$ & $4-3$ & 346998.3360 & 41.6 & $3.27 \times 10^{-3}$ & $0.56(0.01)$ & $10.7(0.1)$ & $2.4(0.1)$ & 0.22 \\
$\mathrm{HOC}^{+}$ & $4-3$ & 357922.0140 & 42.9 & $1.82 \times 10^{-3}$ & $0.21(0.01)$ & $10.6(0.4)$ & $2.8(0.1)$ & 0.07 \\
$\mathrm{SH}^{+}$ & $1_{0}-0_{1} 1 / 2-3 / 2$ & 345944.4205 & 11.6 & $2.30 \times 10^{-4}$ & $0.30(0.01)$ & $10.6(0.2)$ & $2.7(0.1)$ & 0.10 \\
$\mathrm{SO}^{+}$ & $15 / 2^{-}-13 / 2^{+}$ & 347740.0110 & 70.1 & $1.19 \times 10^{-3}$ & $0.36(0.01)$ & $10.6(0.1)$ & $2.1(0.1)$ & 0.16 \\
$\mathrm{SO}$ & $8-78$ & 346528.5280 & 78.8 & $5.38 \times 10^{-4}$ & $3.50(0.08)$ & $10.3(0.2)$ & $1.8(0.1)$ & 1.81 \\
\hline
\end{tabular}

Notes. Velocity-integrated line intensities, LSR velocity of the line emission peaks, line-widths and line temperature peaks obtained by a Gaussian fitting procedure. Parentheses indicate the fit uncertainty.

\section{Appendix B: Rotational population diagrams and column densities}
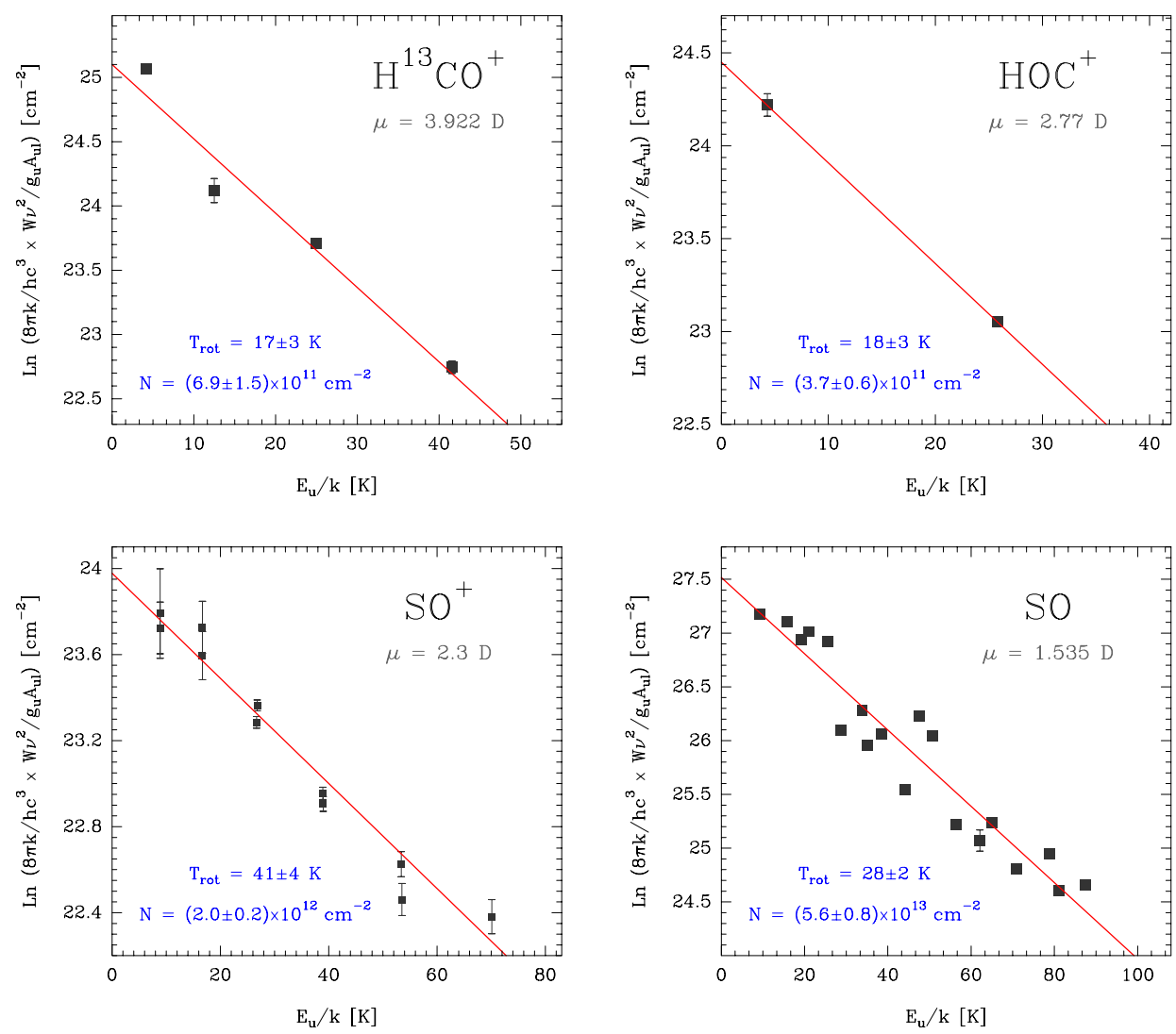

Fig. B.1. Rotational diagrams obtained from IRAM $30 \mathrm{~m}$ observations toward the line survey position (Cuadrado et al. 2015) assuming optically thin emission and uniform beam filling. Fitted values of $T_{\text {rot }}$, column density, and their respective uncertainties are indicated.

Table B.1. Rotational temperatures $\left(T_{\text {rot }}\right)$ and column densities $(N)$ inferred from IRAM $30 \mathrm{~m}$ observations toward the line survey position.

\begin{tabular}{|c|c|c|c|c|}
\hline & \multicolumn{2}{|c|}{ Uniform beam filling } & \multicolumn{2}{|c|}{$10^{\prime \prime}$-width filament } \\
\hline & $\begin{array}{l}T_{\text {rot }} \\
{[\mathrm{K}]}\end{array}$ & $\begin{array}{c}N(\mathrm{X}) \\
{\left[\mathrm{cm}^{-2}\right]}\end{array}$ & $\begin{array}{l}T_{\text {rot }} \\
{[\mathrm{K}]}\end{array}$ & $\begin{array}{l}N(\mathrm{X}) \\
{\left[\mathrm{cm}^{-2}\right]}\end{array}$ \\
\hline $\mathrm{H}^{13} \mathrm{CO}^{+}$ & $17 \pm 3$ & $(6.9 \pm 1.5) \times 10^{11}$ & $13 \pm 2$ & $(1.4 \pm 0.5) \times 10^{12}$ \\
\hline $\mathrm{HOC}^{+}$ & $18 \pm 3$ & $(3.7 \pm 0.6) \times 10^{11}$ & $11 \pm 2$ & $(7.8 \pm 1.2) \times 10^{11}$ \\
\hline $\mathrm{SO}^{+}$ & $41 \pm 4$ & $(2.0 \pm 0.2) \times 10^{12}$ & $29 \pm 3$ & $(3.1 \pm 0.5) \times 10^{12}$ \\
\hline SO & $28 \pm 2$ & $(5.6 \pm 0.8) \times 10^{13}$ & $22 \pm 2$ & $(1.1 \pm 0.2) \times 10^{14}$ \\
\hline $\mathrm{SH}^{+}$ & & & $\sim 10$ & $(1.5 \pm 0.5) \times 10^{13}$ \\
\hline
\end{tabular}




\section{Appendix C: Chemical destruction timescales}

In this Appendix we use the rates of the chemical reactions (included in our PDR model) that lead to the destruction of reactive ions, to compute their characteristic destruction timescales in the Orion Bar. For simplicity we adopt $T_{\mathrm{k}}=T_{\mathrm{e}}=300 \mathrm{~K}$.

The destruction timescales of $\mathrm{CH}^{+}$by reactive collisions with $\mathrm{H}_{2}$ and $\mathrm{H}$ are $\tau_{\mathrm{CH}^{+}}\left(\mathrm{H}_{2}\right) \simeq 4.6 \mathrm{~h}\left(10^{5} \mathrm{~cm}^{-3} / n_{\mathrm{H}}\right) f_{\mathrm{H}_{2}}^{-1}$ and $\tau_{\mathrm{CH}^{+}}(\mathrm{H}) \simeq 3.7 \mathrm{~h}\left(10^{5} \mathrm{~cm}^{-3} / n_{\mathrm{H}}\right)\left(1-f_{\mathrm{H}_{2}}\right)^{-1}$, respectively. In these relations, $f_{\mathrm{H}_{2}}=2 n\left(\mathrm{H}_{2}\right) /\left[n(\mathrm{H})+2 n\left(\mathrm{H}_{2}\right)\right]$ is the molecular gas fraction $\left(<1\right.$ close to the DF of PDRs). The $\mathrm{CH}^{+}$ destruction timescale by dissociative recombination is slower, $\tau_{\mathrm{CH}^{+}}\left(\mathrm{e}^{-}\right) \simeq 185 \mathrm{~h}\left(10^{5} \mathrm{~cm}^{-3} / n_{\mathrm{H}}\right)\left(10^{-4} / x_{\mathrm{e}}\right)$.

On the other hand, $\mathrm{SH}^{+}$slowly reacts with $\mathrm{H}_{2}$ (the reaction is very endothermic). The relevant destruction timescales are $\tau_{\mathrm{SH}^{+}}(\mathrm{H}) \simeq 25 \mathrm{~h}\left(10^{5} \mathrm{~cm}^{-3} / n_{\mathrm{H}}\right)\left(1-f_{\mathrm{H}_{2}}\right)^{-1}$ and $\tau_{\mathrm{SH}^{+}}\left(\mathrm{e}^{-}\right) \simeq 111 \mathrm{~h}$ $\left(10^{5} \mathrm{~cm}^{-3} / n_{\mathrm{H}}\right)\left(10^{-4} / x_{\mathrm{e}}\right)$.

For $\mathrm{HOC}^{+}$we derive $\tau_{\mathrm{HOC}^{+}}\left(\mathrm{H}_{2}\right) \simeq 14.6 \mathrm{~h}\left(10^{5} \mathrm{~cm}^{-3} / n_{\mathrm{H}}\right) f_{\mathrm{H}_{2}}^{-1}$ and $\tau_{\mathrm{HOC}^{+}}\left(\mathrm{e}^{-}\right) \simeq 252 \mathrm{~h}\left(10^{5} \mathrm{~cm}^{-3} / n_{\mathrm{H}}\right)\left(10^{-4} / x_{\mathrm{e}}\right)$.

$\mathrm{SO}^{+}$lifetime is longer. The reaction of $\mathrm{SO}^{+}$with $\mathrm{H}$ is very endothermic. Close to the $\mathrm{DF}, \mathrm{SO}^{+}$destruction is dominated by dissociative recombination, with $\tau_{\mathrm{SO}^{+}}\left(\mathrm{e}^{-}\right) \simeq$ $139 \mathrm{~h}\left(10^{5} \mathrm{~cm}^{-3} / n_{\mathrm{H}}\right)\left(10^{-4} / x_{\mathrm{e}}\right)$, and by photodissociation, with

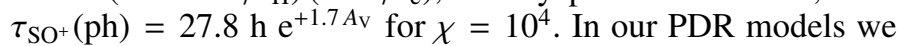
assume $\kappa_{\mathrm{SO}^{+}}(\mathrm{ph})=\chi \times 10^{-9} \mathrm{e}^{-1.7 \mathrm{~A}_{\mathrm{V}}}\left(\mathrm{s}^{-1}\right)$, which is $\simeq 1000, \simeq 10$, $\simeq 5$, and $\simeq 4$ times faster than the adopted $\kappa_{\mathrm{HCO}^{+}}(\mathrm{ph}), \kappa_{\mathrm{CH}^{+}}(\mathrm{ph})$, $\kappa_{\mathrm{OH}}(\mathrm{ph})$, and $\kappa_{\mathrm{SH}^{+}}(\mathrm{ph})$ photodissociation rates, respectively (at $\left.A_{\mathrm{V}}=1\right)$. A summary of the relevant destruction timescales is presented in Table D.1.

\section{Appendix D: Collisional and radiative excitation of $\mathrm{H}^{13} \mathrm{CO}^{+}, \mathrm{HOC}^{+}, \mathrm{SH}^{+}$, and $\mathrm{SO}^{+}$}

To estimate the physical conditions of the reactive ions emitting gas, we solved the statistical equilibrium equations and radiative transfer using a non-local-thermodynamic equilibrium code (Goicoechea et al. 2006). In PDRs, the high electron density (up to $n\left(\mathrm{e}^{-}\right) \simeq x\left(\mathrm{C}^{+}\right) n_{\mathrm{H}} \simeq 10^{-4} n_{\mathrm{H}}$ for standard cosmic ray ionization rates) plays an important role in the collisional excitation of molecular ions and competes with collisions with $\mathrm{H}_{2}$ and $\mathrm{H}$. This is because the associated collisional excitation rate coefficients $\gamma_{\mathrm{lu}}(e)\left(\mathrm{cm}^{3} \mathrm{~s}^{-1}\right)$ can be large, up to $10^{4}$ times $\gamma_{\mathrm{lu}}\left(\mathrm{H}_{2}\right)$, and thus $\mathrm{H}_{2}$ and $e$ collisional rates $\left(\mathrm{s}^{-1}\right)$ become comparable if the ionization fraction $n\left(\mathrm{e}^{-}\right) / n_{\mathrm{H}}$ is high, $x_{\mathrm{e}} \simeq 10^{-5}-10^{-4}$. Here we used published (or estimated by us) inelastic collisional rate coefficients ${ }^{1}$,

${ }_{1-\text { For } \mathrm{H}^{13} \mathrm{CO}^{+} \text {and } \mathrm{HOC}^{+}}\left({ }^{1} \Sigma^{-}\right.$ground electronic states) we used $\mathrm{HCO}^{+}-\mathrm{H}_{2}$ de-excitation rates of Flower (1999), and specific $\mathrm{HCO}^{+}-\mathrm{e}^{-}$ and $\mathrm{HOC}^{+}-\mathrm{e}^{-}$de-excitation rates (presented in Fuente et al. 2008, and references therein). We computed the respective collisional excitation rates through detailed balance.

- For $\mathrm{SH}^{+}\left({ }^{3} \Sigma^{-}\right)$there are no published collisional rate coefficients. For $\mathrm{SH}^{+}-\mathrm{H}_{2}$ we simply scaled radiative rates (J. Black, priv. comm.) for a $10-5000 \mathrm{~K}$ temperature range. For $\mathrm{SH}^{+}-\mathrm{e}^{-}$collisions we used rate coefficients calculated in the Coulomb-Born approximation for the $10-1000 \mathrm{~K}$ temperature range (J. Black, priv. comm.).

- For $\mathrm{SO}^{+}\left({ }^{2} \Pi\right)$ there are no published collisional rates. Here we modeled rotational levels in the ${ }^{2} \Pi_{1 / 2}$ ladder only (the ${ }^{2} \Pi_{3 / 2}$ ladder lies $525 \mathrm{~K}$ above the ground, much higher than the $T_{\text {rot }}\left(\mathrm{SO}^{+}\right)$inferred from our observations) and neglected $\Lambda$-doubling transitions. For $\mathrm{SO}^{+}-\mathrm{H}_{2}$, we used CS-He rates of Lique et al. (2006) (CS has a similar molecular weight and dipole moment: 44 and $2.0 \mathrm{D}$, compared to $\mathrm{SO}^{+}: 48$ and $2.3 \mathrm{D}$. This is the adopted $\mathrm{SO}^{+}$dipole moment after Turner 1996). Rate coefficients were multiplied by 2 to account for the ionic character of $\mathrm{SO}^{+}$ and we adopted $n\left(\mathrm{e}^{-}\right)=10 \mathrm{~cm}^{-3}$ and $n_{\mathrm{H}} \simeq 10^{4}-10^{6} \mathrm{~cm}^{-3}$ in the models, the expected range of densities in the region.

In addition to inelastic collisions, we included radiative excitation by absorption of the $2.7 \mathrm{~K}$ cosmic background and by the (external) dust radiation field in the region. The latter is modelled as a modified blackbody emission with an effective dust temperature of $T_{\mathrm{d}}=50 \mathrm{~K}$, spectral emissivity index of $\beta=1.6$, and a dust opacity $\left(\tau_{\mathrm{d}}\right)$ of $\simeq 0.03$ at a reference wavelength of $\lambda=160 \mu \mathrm{m}$. The resulting continuum levels (Fig. D.1) reproduce the far-IR photometric measurements toward the Orion Bar; we refer to Arab et al. (2012). These authors estimated $T_{\mathrm{d}} \simeq 50 \mathrm{~K}$ inside the Bar and $T_{\mathrm{d}} \simeq 70 \mathrm{~K}$ immediately in front. Our calculations include thermal, turbulent, and line opacity broadening. The nonthermal velocity dispersion that reproduces the observed linewidths is $\sigma \simeq 1 \mathrm{~km} \mathrm{~s}^{-1}$ (with full width at half maximum of $2.355 \cdot \sigma)$.

By varying $N, n_{\mathrm{H}}$ and $T_{\mathrm{k}}$, we tried to reproduce the $\mathrm{H}^{13} \mathrm{CO}^{+}$, $\mathrm{HOC}^{+}, \mathrm{SH}^{+}$, and $\mathrm{SO}^{+}$line intensities observed by ALMA-ACA toward the line survey position as well as the intensities of the other rotational lines detected with the IRAM-30 m telescope (correcting them by a dilution factor that takes into account the beam size at each frequency and an intrinsic $10^{\prime \prime}$-wide Gaussian filament shape of the emission). Our best models fit the intensities by less than a factor of 2 . They also reproduce $( \pm 3 \mathrm{~K})$ the $T_{\text {rot }}$ inferred from the rotational population diagrams for a $10^{\prime \prime}$-wide filament (see Table B.1). For $\mathrm{H}^{13} \mathrm{CO}^{+}, \mathrm{HOC}^{+}$, and $\mathrm{SO}^{+}$, we obtain $n\left(\mathrm{H}_{2}\right) \simeq 10^{5} \mathrm{~cm}^{-3}, n\left(\mathrm{e}^{-}\right)=10 \mathrm{~cm}^{-3}$, and $T_{\mathrm{k}} \gtrsim 200 \mathrm{~K}$. These conditions agree with those inferred by Nagy et al. (2017) for the $\mathrm{H}^{12} \mathrm{CO}^{+}$rotationally excited emission $(J=6-5$ to $11-10)$ observed by Herschel/HIFI.

For $\mathrm{SH}^{+}$, we reproduce the line intensities observed by ALMA-ACA at $\sim 345 \mathrm{GHz}$, and by Herschel/HIFI at $\sim 526 \mathrm{GHz}$ Nagy et al. (2013; significantly diluted in the large HIFI beam according to our ALMA-ACA images), if the gas is an order of magnitude denser, $\sim 10^{6} \mathrm{~cm}^{-3}$ (similar to Nagy et al. 2013 as they used the same estimated collisional rates).

\section{D.1. Radiative pumping rates $\left(\mathrm{CH}^{+}\right.$vs. $\left.\mathrm{HOC}^{+}\right)$}

To support our interpretation, here we compare the collisional and radiative pumping rates of $\mathrm{CH}^{+}$and $\mathrm{HOC}^{+}$rotational lines with their chemical destruction timescales. The inelastic collisional excitation rate $\left(C_{\mathrm{lu}}\right)$ is given by

$C_{\mathrm{lu}}=n\left(\mathrm{H}_{2}\right) \gamma_{\mathrm{lu}}\left(\mathrm{H}_{2}\right)+n\left(\mathrm{e}^{-}\right) \gamma_{\mathrm{lu}}\left(\mathrm{e}^{-}\right) \quad\left[\mathrm{s}^{-1}\right]$,

where we assume that $\mathrm{H}_{2}$ and $\mathrm{e}^{-}$are the only collisional partners. The upward excitation collisional coefficients $\gamma_{\mathrm{lu}}\left(\mathrm{cm}^{3} \mathrm{~s}^{-1}\right)$ are computed from the de-excitation coefficients by detailed balance

$\gamma_{\mathrm{lu}}=\gamma_{\mathrm{ul}} \frac{g_{u}}{g_{l}} \mathrm{e}^{-T^{*} / T_{\mathrm{k}}} \quad\left[\mathrm{cm}^{3} \mathrm{~s}^{-1}\right]$

where $T^{*}=h v / k$ is the equivalent temperature at the frequency $v$ of the transition. The continuum energy density in the cloud at a given frequency is

$U^{\text {Dust }+\mathrm{CMB}}=\beta\left[U\left(T_{\mathrm{d}}\right)+U\left(T_{\mathrm{cmb}}\right)\right]$,

where $U\left(T_{\mathrm{d}}\right)$ is the contribution from the external dust radiation field, and $U\left(T_{\mathrm{cmb}}=2.73 \mathrm{~K}\right)$ is the cosmic background. $\beta$ is the

For $\mathrm{SO}^{+}-\mathrm{e}^{-}$, we used rate coefficients calculated in the Coulomb-Born approximation for the $10-1000 \mathrm{~K}$ range (J. Black, priv. comm.). 


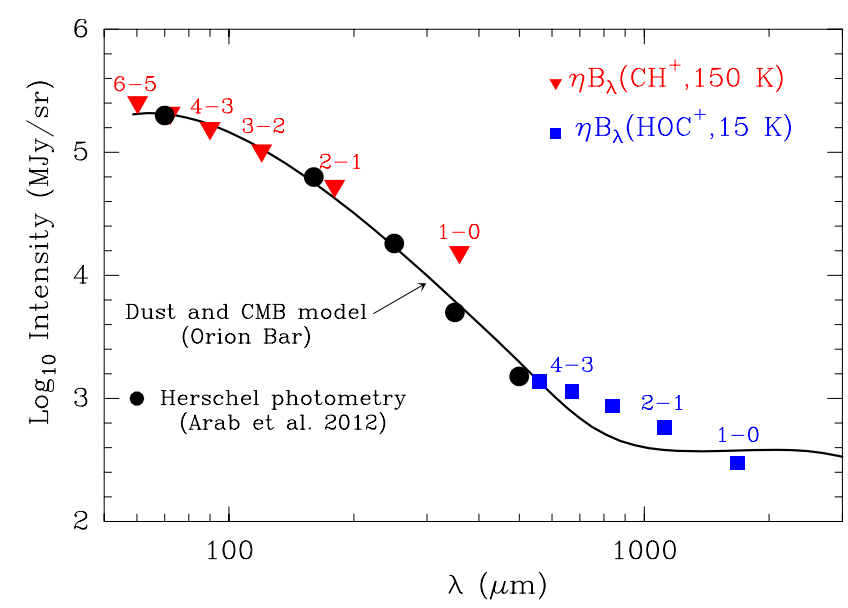

Fig. D.1. Dust continuum emission (with $T_{\mathrm{d}}=50 \mathrm{~K}$ ) and cosmic millimeter background model used in the excitation calculation. Black circles show Herschel's photometric observations toward the Orion Bar. Red triangles show the wavelength position of $\mathrm{CH}^{+}$rotational lines. Their intensity scale is that of a black body at $150 \mathrm{~K}\left(\approx T_{\text {rot }}^{\mathrm{PACS}}\right)$ scaled by an arbitrary dilution factor $\eta$. Blue squares show the position of $\mathrm{HOC}^{+}$ rotational lines. Their intensity scale is that of a black body at $15 \mathrm{~K}$ $\left(\approx T_{\text {rot }}^{\mathrm{IRAM} 30 \mathrm{~m}}\right)$ scaled by a different arbitrary dilution factor.

photon escape probability that tends to 1 if the line opacity tends to 0 . The radiative pumping rate can be written as

$B_{\mathrm{lu}} U^{\text {Dust }+\mathrm{CMB}}=\beta A_{\mathrm{ul}}\left[\frac{1-\mathrm{e}^{-\tau_{\mathrm{d}}}}{\mathrm{e}^{T^{*} / T_{\mathrm{d}}}-1}+\frac{1}{\mathrm{e}^{T^{*} / T_{\mathrm{cmb}}}-1}\right] \quad\left[\mathrm{s}^{-1}\right]$,

where $B_{\mathrm{lu}}$ and $A_{\mathrm{ul}}$ are the Einstein coefficients for stimulated absorption and for spontaneous emission, respectively. As an example of the excitation differences between $\mathrm{CH}^{+}$and the heavier reactive ions, we note that the $\mathrm{CH}^{+} 4-3$ line lies at a far-IR wavelength $(\lambda \simeq 90 \mu \mathrm{m})$ where the intensity of the continuum emission toward the Orion Bar is $\gtrsim 200$ times stronger than that at the submm HOC ${ }^{+} 4-3$ line wavelength $(\lambda \simeq 837 \mu \mathrm{m}$; see Fig. D.1).

We adopt $n\left(\mathrm{H}_{2}\right)=10^{5} \mathrm{~cm}^{-3}, n\left(\mathrm{e}^{-}\right)=10 \mathrm{~cm}^{-3}, T_{\mathrm{k}}=T_{\mathrm{e}}=$ $200 \mathrm{~K}$, and our model of the continuum emission (Fig. D.1). With these parameters, using the appropriate collisional rate coefficients (see Nagy et al. 2013; Godard \& Cernicharo 2013, for detailed $\mathrm{CH}^{+}$excitation models), and in the $\beta=1$ limit, we compute the following collisional and radiative pumping rates

$C_{01}\left(\mathrm{CH}^{+}\right)=3.7 \times 10^{-5} \mathrm{~s}^{-1} \quad(\tau \simeq 7.6 \mathrm{~h})$,

$B_{01} U^{\text {Dust+CMB }}\left(\mathrm{CH}^{+}\right)=1.3 \times 10^{-4} \mathrm{~s}^{-1} \quad(\tau \simeq 2.2 \mathrm{~h})$,

$C_{34}\left(\mathrm{CH}^{+}\right)=5.3 \times 10^{-6} \mathrm{~s}^{-1} \quad(\tau \simeq 52.7 \mathrm{~h})$,

$B_{34} U^{\text {Dust }+\mathrm{CMB}}\left(\mathrm{CH}^{+}\right)=2.1 \times 10^{-3} \mathrm{~s}^{-1} \quad(\tau \simeq 0.1 \mathrm{~h})$,

for $\mathrm{CH}^{+}$, and

$C_{01}\left(\mathrm{HOC}^{+}\right)=1.2 \times 10^{-4} \mathrm{~s}^{-1} \quad(\tau \simeq 2.3 \mathrm{~h})$,

$B_{01} U^{\text {Dust }+\mathrm{CMB}}\left(\mathrm{HOC}^{+}\right)=1.7 \times 10^{-5} \mathrm{~s}^{-1} \quad(\tau \simeq 16.4 \mathrm{~h})$,

$C_{34}\left(\mathrm{HOC}^{+}\right)=7.9 \times 10^{-5} \mathrm{~s}^{-1} \quad(\tau \simeq 3.5 \mathrm{~h})$,

$B_{34} U^{\text {Dust+CMB }}\left(\mathrm{HOC}^{+}\right)=1.6 \times 10^{-5} \mathrm{~s}^{-1} \quad(\tau \simeq 16.9 \mathrm{~h})$,

for $\mathrm{HOC}^{+}$. The quantities in parenthesis $(\tau)$ represent the corresponding timescale for each excitation process (see Table D.2). In the case of reactive molecular ions, these rates compete with their chemical destruction timescales (derived in Appendix C). Adopting a gas density of $10^{5} \mathrm{~cm}^{-3}, f_{\mathrm{H}_{2}}=0.5$ and $x_{\mathrm{e}}=10^{-4}$ for the Orion Bar, the total $\mathrm{CH}^{+}$and $\mathrm{HOC}^{+}$chemical destruction timescales are $\tau_{\mathrm{D}}\left(\mathrm{CH}^{+}\right) \simeq 4 \mathrm{~h}$ and $\tau_{\mathrm{D}}\left(\mathrm{HOC}^{+}\right) \simeq 26 \mathrm{~h}$ (see Table D.1). Comparing $\tau_{\mathrm{D}}\left(\mathrm{CH}^{+}\right)$with the timescales for collisional and radiative excitation shows that $\mathrm{CH}^{+}$molecules are excited by radiation many times during its short lifetime, but not by collisions. Hence, $\mathrm{CH}^{+}$can remain rotationally warm while it emits. Interestingly, the far-IR $\mathrm{CH}^{+}$lines observed by PACS $(\mathrm{J}=$ 3-2 to 6-5, Nagy et al. 2013) follow roughly the same functional shape, $\propto B_{\lambda}\left(T_{\text {rot }}^{\text {PACS }} \simeq 50 \mathrm{~K}\right)$, of the warm dust continuum emission (with $T_{\mathrm{d}} \simeq 50-70 \mathrm{~K}$; Fig. D.1). Because for $\mathrm{CH}^{+}$radiative processes are faster than collisional processes (Table D.2), $\mathrm{CH}^{+}$molecules might have equilibrated with the dust radiation field they absorb (Black 1998). However, Godard \& Cernicharo (2013) conclude that the high- $J \mathrm{CH}^{+}$lines in the Bar are mostly driven by formation pumping; thus, naturally producing warm rotational temperatures. On the other hand, comparing $\tau_{\mathrm{D}}\left(\mathrm{HOC}^{+}\right)$with the representative timescales for collisional and radiative excitation shows that $\mathrm{HOC}^{+}$molecules (and likely the other heavier ions as well) are excited by collisions several times during their lifetime. Inelastic collisions thus can drive their rotational populations to lower $T_{\text {rot }}$ relatively fast. 
J. R. Goicoechea et al.: Spatially resolved images of reactive ions in the Orion Bar

Table D.1. Timescales, in hours, for chemical destruction by reactive collisions with $\mathrm{H}_{2}, \mathrm{H}, \mathrm{e}^{-}$and by FUV photodissociation.

\begin{tabular}{|c|c|c|c|c|}
\hline Ion & $\tau\left(\mathrm{H}_{2}\right)^{a}$ & $\tau(\mathrm{H})^{a}$ & $\tau\left(\mathrm{e}^{-}\right)^{a}$ & $\tau$ (photodiss. $^{b}$ \\
\hline $\begin{array}{l}\mathrm{CH}^{+} \\
\tau_{\mathrm{D}} \approx 4 \mathrm{~h}^{c}\end{array}$ & $4.6 \mathrm{~h}\left(10^{5} \mathrm{~cm}^{-3} / n_{\mathrm{H}}\right) f_{\mathrm{H}_{2}}^{-1}$ & $3.7 \mathrm{~h}\left(10^{5} \mathrm{~cm}^{-3} / n_{\mathrm{H}}\right)\left(1-f_{\mathrm{H}_{2}}\right)^{-1}$ & $185 \mathrm{~h}\left(10^{5} \mathrm{~cm}^{-3} / n_{\mathrm{H}}\right)\left(10^{-4} / x_{\mathrm{e}}\right)$ & $84.2 \mathrm{~h} \mathrm{e}^{+2.94 A_{V}}$ \\
\hline $\begin{array}{l}\mathrm{HOC}^{+} \\
\tau_{\mathrm{D}} \approx 26 \mathrm{~h}^{c}\end{array}$ & $14.6 \mathrm{~h}\left(10^{5} \mathrm{~cm}^{-3} / n_{\mathrm{H}}\right) f_{\mathrm{H}_{2}}^{-1}$ & - & $252 \mathrm{~h}\left(10^{5} \mathrm{~cm}^{-3} / n_{\mathrm{H}}\right)\left(10^{-4} / x_{\mathrm{e}}\right)$ & $5144 \mathrm{~h} \mathrm{e}^{+3.32 A_{V}}$ \\
\hline $\begin{array}{l}\mathrm{SH}^{+} \\
\tau_{\mathrm{D}} \approx 46 \mathrm{~h}^{c}\end{array}$ & - & $25 \mathrm{~h}\left(10^{5} \mathrm{~cm}^{-3} / n_{\mathrm{H}}\right)\left(1-f_{\mathrm{H}_{2}}\right)^{-1}$ & $111 \mathrm{~h}\left(10^{5} \mathrm{~cm}^{-3} / n_{\mathrm{H}}\right)\left(10^{-4} / x_{\mathrm{e}}\right)$ & $111 \mathrm{he}^{+1.66 A_{V}}$ \\
\hline $\begin{array}{l}\mathrm{SO}^{+} \\
\tau_{\mathrm{D}} \approx 73 \mathrm{~h}^{c}\end{array}$ & - & - & $139 \mathrm{~h}\left(10^{5} \mathrm{~cm}^{-3} / n_{\mathrm{H}}\right)\left(10^{-4} / x_{\mathrm{e}}\right)$ & $27.8 \mathrm{he}^{+1.70 A_{V}}$ \\
\hline
\end{tabular}

Notes. ${ }^{(a)}$ Assuming $T_{\mathrm{k}}=T_{\mathrm{e}}=300 \mathrm{~K}$. ${ }^{(b)}$ For a FUV-radiation field of $\chi=10^{4} .{ }^{(c)}$ Total destruction timescale $\left(\tau_{\mathrm{D}}\right)$ at $A_{V}=1$ assuming $n_{\mathrm{H}}=10^{5} \mathrm{~cm}^{-3}$ and $f_{\mathrm{H}_{2}}=0.5$. For $\mathrm{CH}^{+}\left(\mathrm{HOC}^{+}\right), \tau_{\mathrm{D}}$ is shorter (longer) than the timescale for non-reactive collisions in their low-lying rotational levels (see Table D.2.).

Table D.2. Representative timescales for non-reactive inelastic collisions and for radiative excitations of $\mathrm{CH}^{+}$and $\mathrm{HOC}^{+}$low-lying levels.

\begin{tabular}{ccccc}
\hline \hline Ion & $\tau_{0 \rightarrow 1}(\text { coll. })^{a}$ & $\tau_{3 \rightarrow 4}(\text { coll. })^{a}$ & $\tau_{0 \rightarrow 1}(\mathrm{rad} .)^{b}$ & $\tau_{3 \rightarrow 4}(\text { rad. })^{b}$ \\
\hline $\mathrm{CH}^{+} J_{1} \rightarrow J_{\mathrm{u}}$ & $7.6 \mathrm{~h}$ & $52.7 \mathrm{~h}$ & $2.2 \mathrm{~h}$ & $0.1 \mathrm{~h}$ \\
$\mathrm{HOC}^{+} J_{1} \rightarrow J_{\mathrm{u}}$ & $2.3 \mathrm{~h}$ & $3.5 \mathrm{~h}$ & $16.4 \mathrm{~h}$ & $16.9 \mathrm{~h}$ \\
\hline
\end{tabular}

Notes. ${ }^{(a)}$ Adopting $n\left(\mathrm{H}_{2}\right)=10^{5} \mathrm{~cm}^{-3}$ and $n\left(\mathrm{e}^{-}\right)=10 \mathrm{~cm}^{-3}$. ${ }^{(b)}$ Using our model of the continuum emission in the Orion Bar (see Fig. D.1). 